BULL. AUSTRAL. MATH. SOC.

$90020,90 C 50$

VOL. $25(1982), 159-160$.

\title{
A QUADRATIC PROGRAMMING ALGORITHM \\ FOR GEOPHYSICAL GRAVITY INVERSION \\ AND OTHER APPLICATIONS
}

Nigel JOHN Fisher

The geophysical gravity method is described and the corresponding inverse problem stated as an integral equation. A survey of previous work associated with gravity data inversion is given followed by an account of the inherent difficulties associated with gravity inversion; this is done by a discussion of the integral equation already given, the inversion of which constitutes an ill-posed problem. The integral equation gives rise to an ill-conditioned system of linear equations; existing methods for the solutions of such systems are reviewed. Then follows an account of the previous use of quadratic programming techniques in the solution of illposed problems.

A new approach to the inverse gravity problem is described, via formulation as a problem in quadratic programming. This quadratic programing problem is stabilised (a well-posed problem is created from the previously ill-posed problem) by the use of both linear constraints and damping. Two examples of the method, one with field data and one with synthetic data, are presented, the solutions being effected via an existing quadratic programming algorithm. This algorithm, however, is unable to handle certain kinds of a priori information associated with the gravity problem and a new algorithm, based on the existing one, is developed to handle the additional constraints. The new algorithm is then run on the two sets of gravity data, having first been tested on a range of other

Received 7 October 1981. Thesis submitted to the University of Queensland February 1981. Degree approved July 1981. Supervisors: Dr L.E. Howard, Dr J.N. Holt. 
problems.

The quadratic programing method of solution for the gravity problem as it is posed is compared with other methods of solution, the comparison being favourable to the quadratic programming approach. The new algorithm is applied to a problem of data approximation and a variation of the new algorithm is applied to a problem in mathematical biology. The thesis concludes with suggestions for future work.

Department of Mathematics and Computer Science, Queensland Institute of Technology, PO Box 246, North Quay, Queensland 4000, Australia. 